\title{
Editorial
}

\section{Beyond HLA-B27: Changing Paradigms in the Genetics of Ankylosing Spondylitis}

I urge you to engrave this on the template of your memories: There are thousands of diseases in this world, but medical science only has an empirical cure for twenty-six of them. The rest is ... guesswork.

Ardent followers of the novelist Erich Segal may identify the above quote from the famous novel doctors, where a professor tells the fresh-faced medical students, much to their bewilderment, that medicine is mostly intelligent guesswork. Whereas this quote may raise quite a few eyebrows, the reader would agree that the etiology of most systemic diseases is multifactorial and our understanding of the disease mechanisms continues to evolve. In the quest to understand the pathogenesis of diseases, perhaps the most widely used methodology is the study of disease associations. Indeed, some of the associations have immensely improved our understanding and consequently the treatment of diseases; famous examples include association of smoking with lung cancer, human papillomavirus with cervical cancer, and Helicobacter pylori with acid peptic disease.

In 1973, Brewerton et $\mathrm{al}^{1}$ described the association of HLA-B27 with ankylosing spondylitis (AS). This spurred research looking at the role of HLA-B27 and other major histocompatibility complex (MHC) molecules in the pathogenesis of AS. It is believed that the prevalence of HLA-B27 and worldwide distribution of AS are closely linked. However, some regions show disproportionately low prevalence of the disease despite high prevalence of HLA-B27 in the population. This discrepancy is mainly due to the existence of variable HLA-B27 subtypes among different populations. Till date, more than 180 different HLA-B27 subtypes are known (http:/ / hla.alleles.org/alleles/class1.html), with some being strongly associated (HLA-B*2704 and HLA-B*2705) and others weakly associated (HLA-B*2706 and HLA-B ${ }^{*} 2709$ ) with the disease. ${ }^{2}$ However, even after 4 decades since it was reported, the exact mechanisms via which HLA-B27 contributes to the pathogenesis of AS remain enigmatic, although several hypotheses, including arthritogenic peptide theory, unfolded protein stress response, misfolding, refolding, autodisplay, and cross-display of HLA-B27 molecules, have been proposed., ${ }^{3,4}$ Furthermore, the role of HLA-B27 as a standalone diagnostic marker in AS remains uncertain owing to the fact that only 1 to $2 \%$ of HLA-B27 positive individuals develop AS. ${ }^{2,5}$ Besides HLA-B27, certain other HLA class I genes have also been implicated in AS causation like HLA-A*02:01, HLA-B*13:02, HLA-B*40:01, HLA-B*40:02, HLA-B ${ }^{*} 47: 01$, and HLA-B*51:01. ${ }^{6}$ It may intrigue the reader that although most autoimmune diseases have been linked to HLA class II molecules, the association of AS with these molecules has been established only recently. Recent research has linked AS with some HLA-DR alleles like the HLA-DRB1, HLA-DRB1*01:03, and HLA-DPB1. ${ }^{6}$ Furthermore, several genome-wide association studies have established the polygenic nature of AS and have revealed that HLA-B27 accounts for only one-third of the genetic basis of AS. This finding has encouraged researchers to study the role of non-MHC genes in the pathogenesis of AS. More than 100 loci have been studied in this context. IL-23R, ERAP1 and 2, IL-7R, IL-1R2-R1, IL-12B, and RUNX3 are among the most commonly associated non-MHC genes in different populations. ${ }^{2} \mathrm{Of}$ all these, perhaps the strongest associations have been described with the single nucleotide polymorphisms (SNPs) in IL-23R and ERAP genes.

IL-23R gene is located on chromosome 1p31.3. It encodes the IL-23R subunit of the heterodimeric IL-23 receptor and acts through the IL-23/IL-17 axis. Genome-wide association studies have shown the association of several IL-23R SNPs with AS, thereby indicating a definite role of IL-17 producing IL-23 responsive cells in the disease pathogenesis. Multiple studies in different populations have confirmed this association. This makes IL-23R, a potential therapeutic target in patients with AS.

ERAP1 and 2 are closely located genes on chromosome 5q15. These encode the corresponding aminopeptidases, which regulate the trimming of peptides in endoplasmic reticulum for presentation to class I MHC molecules. Several studies have shown that SNPs in ERAP1 are associated with HLA-B*27 and HLA$B^{*} 40$ positive AS, but not with HLA-B*27/B*40 doublenegative AS. The SNPs in ERAP2 are associated both with HLA-B*27positive and negative AS. ${ }^{2}$

To conclude, the pathogenesis of AS continues to be a mystery, and our understanding of its genetics is evolving. However, it is time to look beyond the conventional teaching of HLA-B27 as the only genetic factor 
in AS. Ongoing research holds promise, not only in unraveling its etiopathogenesis, but also for identification of newer specific diagnostic biomarkers, potential therapeutic molecular targets, and development of targeted therapies.

\section{REFERENCES}

1. Brewerton DA, Hart FD, Nicholls A, Caffrey M, James DC, Sturrock RD. Ankylosing spondylitis and HL-A 27. Lancet 1973 Apr;1(7809):904-907.

2. Brown MA, Kenna T, Wordsworth BP. Genetics of ankylosing spondylitis - insights into pathogenesis. Nat Rev Rheumatol 2016 Feb;12(2):81-91.

3. Luthra-Guptasarma M, Singh B. HLA-B27 lacking associated beta2-microglobulin rearranges to auto-display or cross-display residues 169-181: a novel molecular mechanism for spondyloarthropathies. FEBS Lett 2004 Sep;575(1-3):1-8.

4. Brown MA. Genetics of ankylosing spondylitis. Curr Opin Rheumatol 2010 Mar;22(2):126-132.

5. Reveille JD. The genetic basis of spondyloarthritis. Ann Rheum Dis 2011 Mar;70 (Suppl 1):i44-i50.

6. Cortes A, Pulit SL, Leo PJ, Pointon JJ, Robinson PC, Weisman MH, Ward M, Gensler LS, Zhou X, Garchon HJ. Major histocompatibility complex associations of ankylosing spondylitis are complex and involve further epistasis with ERAP1. Nat Commun 2015 May;6:7146.17 12017

Parikshaa Gupta Senior Research Associate Department of Immunopathology Postgraduate Institute of Medical Education \& Research, Chandigarh, India

Ranjana W Minz

Professor and Head Department of Immunopathology Postgraduate Institute of Medical Education \& Research, Chandigarh, India 